\title{
Uncommon presentation of hypereosinophilic syndrome
}

\author{
J Rajkanna ${ }^{1}$, J Indrakumar², S Kamaladasa², C C Kariyawasan ${ }^{3}$
}

(Index words: hypereosinophilic syndrome, mucosal ulceration)

\section{Introduction}

The hypereosinophilic syndrome is a group of diseases characterised by persistent blood eosinophilia, defined as more than 1500 cells per micro liter [1], with end-organ involvement and no recognised secondary causes such as skin diseases, parasitic infection or allergy (Table). The sustained overproduction of eosinophils causes eosinophilic infiltration and release of mediators causing damage to multiple organs [2]. Cutaneous involvement occurs in more than $50 \%$ of patients, but it usually appears late in the disease and is of less importance than cardiac and other organ involvement [1]. We describe a patient with the hypereosinophilic syndrome (HES) in whom the initial manifestation of the disease was recurrent, severe mucosal ulcers involving the mouth and genitalia.

\section{Case report}

A 39-year old man was well until January 2007 when he developed persistent oral ulcers. Two months later, the oral ulcers increased and he also developed erosive lesions on the glans penis and ulceration of the skin of the scrotum (Figures 1 and 2). Clinical examination of the heart, lungs and abdomen were unremarkable and there were no other skin lesions. His white cell count was $35 \times 10^{9} / 1$ with $54 \%$ eosinophils. He underwent investigations including stool tests for amoebae, ova and cysts, and bone marrow examination which did not show a cause for his illness. He was treated with sequential courses of intravenous antibiotics, albendazole, di-ethyl carbamazine and a short course of steroids without improvement in his symptoms or eosinophil count. He was discharged from hospital and was followed up in the clinic.

In August 2007 he was re-evaluated because of persistent orogenital ulcers and eosinophilia without other skin lesions. His white blood counts at that time were $23 \times 10^{9} / 1$ with $60 \%$ eosinophils. The haemoglobin and platelets were within normal range. Eosinophilic leukaemia (myeloid cells that demonstrate a clonal chromosomal abnormality [4]) was excluded by blood picture, bone

${ }^{1}$ University Medical Unit, Colombo South Teaching Hospital, Kalubowila, and Departments of ${ }^{2}$ Medicine and ${ }^{3}$ Pathology, Faculty of Medical Sciences, University of Sri Jayawardenepura, Nugegoda, Sri Lanka.

Correspondence: JI, e-mail <sathika@sltnet.lk>. Received 29 December 2008 and revised version accepted 16 May 2009. Competing interest: none declared. 
marrow, normal NAP (neutrophil alkaline phosphatase) score and negative BCR-ABL gene (RT-PDR) studies. Multiple skin and mucosal biopsies excluded pemphigus and other skin diseases. The pathergy test, CEA, ANF, PSA, VDRL, skin smears and antibodies for herpes simplex were negative. Ultrasound scan of the abdomen showed mild splenomegaly. 2D echo cardiogram showed normal cardiac structure and function.

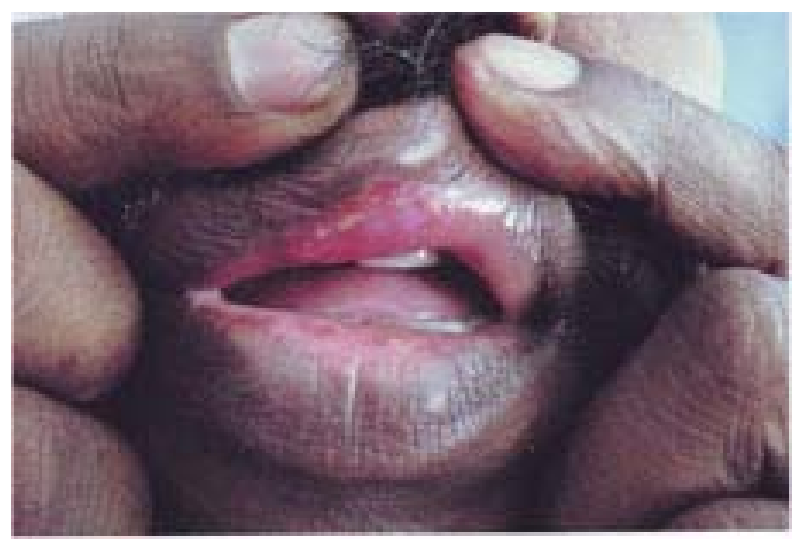

Figure 1

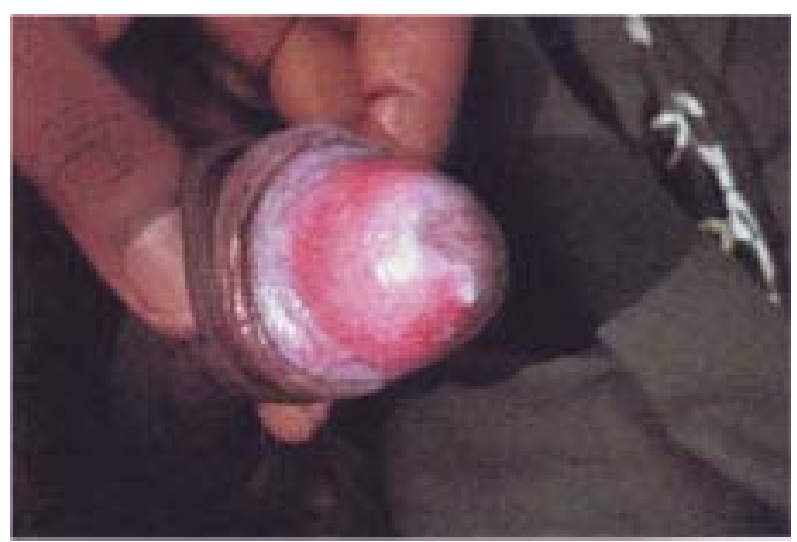

Figure 2

Genetic studies for the mutant gene FIP1L1-PDGFRA (FIP1Like1 Platelet-derived growth factor receptor, alpha polypeptide) was positive. The patient was treated with oral imatinib $100 \mathrm{mg}$ (tyrosine kinase inhibitor) every week. There was a dramatic drop in the white cell count to $6 \times$ $10 \% / 1$ with marked reduction of the eosinophil count to $5 \%$ one week after starting treatment. The orogenital ulcers healed completely within two weeks. The patient is currently being followed up in the clinic.

\section{Discussion}

The hypereosinophilic syndrome is a multisystem disease characterised by infiltration of eosinophils in the bone marrow, heart, and other organs [2]. Cutaneous, and rarely, mucosal involvement occurs, but unlike in our patient they usually appear late in the disease [2]. Mucosal ulcerations can cause significant morbidity and are difficult to treat.

Mucosal ulcerations are a variant presentation that appear to be a marker for a mutation that characterises a subgroup of HES patients [3]. A proportion of HES patients have a mutation involving the PDGFRA and FIP1L1genes on the fourth chromosome, leading to a tyrosine kinase fusion protein. The prevalence of the FIP1L1-PDGFR alpha fusion gene in patients with HES is reported to be $11 \%$ [3]. Its presence indicates responsiveness to imatinib, a tyrosine kinase inhibitor [1].

\section{Table. Diagnostic criteria for hypereosinophilic syndrome [2]}

1. Eosinophil counts $>1.5 \times 10^{9} / 1$ for $<6$ months or $<6$ months with evidence of organ damage

2. Lack of evidence for parasitic, allergic, or other recognised causes of eosinophilia

3. Symptoms and signs of organ-system involvement

\section{References}

1. Leiferman KM, Gleich GJ. Hypereosinophilic syndrome: case presentation and update. Journal of Allergy and Clinical Immunology 2004; 113: 50-8.

2. Brugnoni D, Airo P, Rossi G, et al. A case of hypereosinophilic syndrome is associated with the expansion of a CD3CD4+ T-cell population able to secrete large amounts of interleukin-5. Blood 1996; 87: 1416-22.

3 Jovanovic JV, Scare J, Waghorns K. Low dose imatinib mwsylate leads to rapid induction of major molecular response and achievement of complete molecular remission in FIP1L1-PDGRA positive chronic eosinophilic leukaemia. Blood 2007; 109: 4635-40.

4. Bain B, Pierre R, Imbert M, et al. Chronic eosinophilic leukaemia and the hypereosinophilic syndrome. In: Jaffe ES, Harris NL, Stein H, Vardiman JW, eds. World Health Organization Classification of Tumours: Pathology and Genetics of Tumours of Haematopoietic and Lymphoid Tissues. Lyon, France: IARC Press, 2001: 29-31. 